\title{
Mulher mastectomizada em tratamento quimioterápico: um estudo dos comportamentos na perspectiva do modelo adaptativo de Roy
}

A mastectomized woman submitted to chemotherapy treatment: a behavioral study according to Roy's adaptative model perspectives

\section{Resumo}

A mulher portadora de câncer de mama vivencia conflitos psicológicos e distúrbios emocionais antes do início do tratamento. O bjetivou-se conhecer os estímulos que atuam na mulher mastectomizada em tratamento quimioterápico, identificar os comportamentos desta frente aos estímulos estabelecendo os diagnósticos de enfermagem e elaborar intervenções de enfermagem com vistas a auxiliar a mulher na promoção de respostas adaptativas. 0 estudo de caso foi realizado com uma mulher mastectomizada, inserida no grupo de auto-ajuda à mulher mastectomizada, da Universidade Federal do Ceará, utilizando como referencial teórico o modelo de adaptação de Roy. Os dados foram coletados em quatro visitas domiciliares, por meio de um roteiro de levantamento de dados. A avaliação dos comportamentos e estímulos possibilitou a elaboração dos seguintes diagnósticos: distúrbio no padrão de sono relacionado à dor e modificações no ambiente; dor relacionada a distúrbios secundários ao câncer; nutrição alterada; integridade da pele prejudicada; ansiedade relacionada à ameaça ao autoconceito; distúrbio na imagem corporal relacionado às mudanças na aparência, secundárias à quimioterapia; e isolamento social relacionado à aparência deffigurada e ao medo de rejeição secundários ao câncer. Por meio das intervenções de enfermagem, os principais resultados foram: melhora do problema do sono e da nutrição; utilização de artifícios para melhora da imagem corporal e visitas freqüentes a amigos e familiares. A cliente evoluiu de maneira satisfatória, apresentando gradualmente comportamentos adaptativos. 0 processo de enfermagem de Roy proporcionou a identificação de estímulos, possibilitando o planejamento de intervenções mais específicas.

Palavras-chave: neoplasias mamárias; quimioterapia; diagnóstico de enfermagem; comportamento; adaptação psicológica; relações enfermeiro-paciente.

\footnotetext{
${ }^{1}$ M estra em Enfermagem Clínico-cirúrgica pela U niversidade Federal do C eará. Enfermeira do H ospital D istrital Evandro Ayres deM oura - CE. Enviar correspondência para E.M .M . RuaBom Sucesso 437, Antônio Bezerra; 60356-310 Fortaleza, CE - Brasil. E-mail: elizmelo@mixmail.com

${ }^{2} D$ outora em Enfermagem. Professora Adjunto do D epartamento deEnfermagem eC oordenadora do Programa dePós-G raduação daU niversidade Federal do C eará.

${ }^{3}$ Enfermeira. M estra em Enfermagem C línico-cirúrgica pela U niversidadeFederal do C eará.

${ }^{4}$ Enfermeira. M estra em Enfermagem C línico-cirúrgica pela U niversidade Federal do C eará.

Recebido em outubro de 2000.
} 


\begin{abstract}
A woman with breast cancer experiences psychological conflicts and emotional disturbances before the beginning of the treatment. It was aimed: to know the stimuli that act in the mastectomized woman in chemotherapy treatment; to identify her forms of behavior when facing these simuli, establishing nursing diagnoses; and to elaborate nursing interventions, in order to help the woman in promoting of adaptative responses. This case study was carried out with a mastectomized woman who attended the mastectomized women support group of Ceara's Federal University, using as theoretical reference Roys adaptative model. Data were collected at a form, over four home visits. The behavior and stimuli evaluation pointed to the following diagnoses seep disorder related to pain and environmental modifications, pain related to secondary cancer disorder; altered nutrition; skin damage; anxiety related to the idea of jeopardized self-image; disturbance in the corporal image related to appearance changes secondary to the chemotherapy; and social isolation related to the deformed appearance and to the rejection fear secondary to cancer. After nursing interventions, the main results were: sleeping and nutrition problems improvement; use of artifices due to the corporal image improvement and friends and family frequent visiting. The client has developed in a satisfactory way, presenting adaptative behaviors gradually. Roys nursing process provided the stimuli identification, making possible the planning of more specific interventions.
\end{abstract}

Key words breast neoplasms, chemotherapy; nursing diagnosis; behavior; psychological adaptation; nurse patient relations.

\section{INTRODUÇÃO}

A quimioterapia representa um avanço na cura e controle do câncer, aumentando a expectativa de vida do paciente. É essencial, no entanto, que os profissionais de saúde que atuam nessa área tornem efetiva sua orientação quanto aos objetivos e efeitos colaterais do tratamento quimioterápico, além, sobretudo, de oferecer apoio emocional.

Via de regra, há a combinação de dois ou mais agentes para a obtenção de uma resposta mais eficiente do que se um único agente fosse utilizado. Esta combinação também diminui a incidência e a severidade dos efeitos colaterais, visto que um dos agentes serve para reverter 0 efeito do outro. ${ }^{1}$

Como modalidade de tratamento do câncer de mama, a quimioterapia tem o seu papel estabelecido, seja em caráter neoadjuvante, adjuvante ou paliativo. Enquanto não se consegue reverter o processo biológico que altera o comportamento da célula maligna, a quimioterapia continuará sendo usada como método auxiliar na tentativa de cura ou de aumento de sobrevida em pacientes portadoras de carcinoma mamário. ${ }^{2}$

Vale enfatizar que, frente ao diagnóstico de câncer de mama, a mulher vivencia sentimentos de medo, tristeza e negação, associados à importância da mama como símbolo da feminilidade, mas também devido ao medo quanto aos tratamentos que poderão ser indicados, entre os quais se inclui a quimioterapia.

N esse sentido, a mulher mastectomizada, quando em tratamento quimioterápico, tende a ficar mais sensível e vulnerável aos estímulos que o mesmo provoca, muitas vezes interferindo na promoção de uma resposta eficaz diante desses mesmos estímulos, o que contribui, de forma negativa, para a sua adaptação.

Ressalta-se, no que diz respeito à implementação da assistência de enfermagem, a importância da utilização de um modelo teórico, no caso o M odelo de Adaptação de Roy, pois a quimioterapia gera estímulos, exigindo da mulher uma resposta, que poderá ser tanto adaptativa como ineficaz. Roy e Andrews $^{3}$ descreve a pessoa como um sistema adaptativo, exposto a estímulos que lhe exigem uma resposta, podendo ou não contribuir para a manutenção de sua integridade. Os estímulos são de três tipos: focal, contextual e residual.

Estímulo focal é o estímulo interno ou externo, capaz de confrontar, imediatamente, 
o indivíduo e provocar o comportamento ou resposta. Estímulo contextual representa todos os outros estímulos, internos ou externos, presentes na situação, contribuindo para 0 efeito do estímulo focal. Estímulo residual é definido como fatores internos ou externos que possuem um efeito indeterminado no sistema adaptativo humano. ${ }^{3}$

O s comportamentos da pessoa podem ser observados através dos quatro modos adaptativos: fisiológico, autoconceito, função de papéis e interdependência. 0 modo fisiológico se relaciona às cinco necessidades fisiológicas básicas: oxigenação, nutrição, eliminação, proteção, atividade e repouso. 0 modo de autoconceito enfoca aspectos psicológico e espiritual da pessoa. 0 modo dafunção depapéis enfoca os papéis desempenhados pela pessoa na sociedade, enquanto 0 modo de interdependência é definido como relações estreitas entre as pessoas.

A prática de enfermagem é realizada através do processo de enfermagem. 0 processo de enfermagem de Roy e Andrews ${ }^{4}$ destaca seis fases:

a) avaliação de comportamentos; b) avaliação de estímulos; c) diagnóstico de enfermagem; d) estabelecimento de metas; e) intervenção; f) avaliação.

$\mathrm{N}$ esse sentido, o presente estudo visa conhecer os estímulos que atuam na mulher mastectomizada em tratamento quimioterápico; identificar o seu comportamento frente aos estímulos, estabelecendo os diagnósticos de enfermagem; e elaborar intervenções de enfermagem, com vistas a auxiliá-la na promoção de respostas adaptativas.

\section{MATERIAL E MÉTODOS}

A pesquisa é do tipo estudo de caso, que pode abordar tanto de um caso simples como um complexo. 0 caso deve ser bem delimitado, tendo seus contornos claramente definidos no desenrolar do estudo. ${ }^{5}$

0 trabalho em questão foi realizado com uma mulher mastectomizada, vinculada ao grupo de auto-ajuda, ensino, pesquisa e assistência à mulher mastectomizada (GEPAM ), que funciona no $D$ epartamento de Enfermagem da Universidade Federal do
Ceará, com reuniões semanais, quando são realizadas oficinas educativas, terapias, orientação individual e grupal.

O s dados foram coletados no período de outubro a novembro de 1999, durante quatro visitas domiciliares, quando foi utilizado um roteiro de levantamento de dados, com base na teoria de Adaptação de Roy, elaborado a partir do modelo de Christensen e Kenney. ${ }^{6}$ 0 referido modelo foi adequado à paciente do estudo, enfatizando os comportamentos característicos de sua situação.

Em um primeiro momento, o roteiro prestou-se para avaliar os comportamentos e os estímulos. A partir daí, foram estabelecidos os diagnósticos de enfermagem, relacionando os modos adaptativos, comportamentos e estímulos, 0 que proporcionou 0 estabelecimento de metas e intervenções a fim de promover uma resposta adaptativa por parte da mulher. 0 último momento constou da avaliação das intervenções.

Serviram como referencial para 0 estabelecimento dos diagnósticos, Iyer et al. ${ }^{7}$ os quais colocam que para se chegar ao diagnóstico, deve-se passar anteriormente pela fase de processamento dos dados, constituída por três etapas: a) classificação; b) interpretação e c) confirmação dos dados. À enfermeira compete reunir os dados, identificar indicações, fazer inferências sobre o estado de saúde do cliente e confirmar esses julgamentos com o cliente.

Para a classificação dos dados, as informações foram distribuídas nos modos adaptativos de Roy, permitindo a melhor organização das informações e a focalização dos dados pertinentes às necessidades da cliente. Após este passo, seguiu-se a interpretação dos dados e a identificação dos estímulos focais, contextuais e residuais. $E$, por fim, foram os dados confirmados, através da interação direta com a cliente.

Depois de percorrida a fase de processamento dos dados, foram formulados os diagnósticos de enfermagem, utilizandose como base para o enunciado a taxonomia apresentada por Carpenito, ${ }^{8}$ a qual está centrada na proposta da N AN DA.

Antes da formulação final do diagnóstico de enfermagem, é fundamental que se 
verifique sua exatidão. Esta seria a fase de confirmação, realizada com base nos comportamentos e estímulos avaliados, em associação com a validação junto à cliente. ${ }^{9}$ As reuniões com a orientadora auxiliaram na confirmação, uma vez que nessas oportunidades era discutida a adequação da denominação diagnóstica.

A documentação, que representa a 4 a fase do processo diagnóstico citado por lyer et al.,, foi realizada por meio da elaboração do relatório de estudo, juntamente com 0 registro do plano de cuidados.
D e acordo com o processo de enfermagem proposto no M odelo de Roy, apóso diagnóstico de enfermagem, ter-se-ia como seqüência, 0 estabelecimento de metas de enfermagem, intervenção eavaliação, o que defato aconteceu.

\section{RESULTADOS E DISCUSSÃO}

Avaliação de comportamentos e estímulos

A utilização dos dados coletados, seguindo

o roteiro de levantamento, permitiu a avaliação dos comportamentos da mulher,

Tabela 1. Relação entre o modo adaptivo, os estímulos presentes e os comportamentos associados

\begin{tabular}{|c|c|c|}
\hline Modo Adaptativo & Comportamentos & Estímulos: focais ( $F$ ), contextuais $(\mathrm{C})$ e residuais $(\mathrm{R})$ \\
\hline \multirow{15}{*}{ Fisiológico } & Falta de disposição para atividades diárias & Pouco tempo dedicado ao sono/repouso (F) \\
\hline & \multirow[t]{3}{*}{ Dificuldades para conciliar sono erepouso } & Dores na região torácica posterior (F) \\
\hline & & Dores na região da cirurgia de reconstrução mamária $(\mathrm{F})$ \\
\hline & & Mudança de ambiente (F) \\
\hline & Dores na região torácica posterior & Cirurgia (C) \\
\hline & Dores na região da cirurgia de & Câncer de mama (C) \\
\hline & reconstrução mamária & Crenças e valores sobre vida e morte (R) \\
\hline & \multirow{4}{*}{ Redução no peso } & Cirurgia ainda não cicatrizada (F) \\
\hline & & Quimioterapia (C) \\
\hline & & Câncer de mama (C) \\
\hline & & Enjôo e náuseas (F) \\
\hline & Enjôo e náuseas & Anorexia (F) \\
\hline & Anorexia & Mudanças na dieta (F) \\
\hline & Pele ressecada & Quimioterapia (C) \\
\hline & Couro cabeludo ressecado, com presenças de bolhas e prurido & Efeitos adversos relacionados ao tratamento (F) \\
\hline \multirow{7}{*}{$\begin{array}{l}\text { Autoconceito } \\
\text { Self físico }\end{array}$} & Tristeza e vergonha com relação ao corpo & Cirurgia de reconstrução mamária não concluída (F) \\
\hline & Mudança no relacionamento com 0 & Vergonha do corpo (F) \\
\hline & companheiro & Mastectomia e quimioterapia (C) \\
\hline & \multirow[t]{3}{*}{ Isolamento social } & Vergonha (queda do cabelo) (F) \\
\hline & & Quimioterapia (C) \\
\hline & & Conceitos pessoais relativos a aparência física (R) \\
\hline & Dependência & Mobilidade física prejudicada/parou de trabalhar $(F)$ \\
\hline Self pessoal & Tristeza e auto-estima afetada & Falta de demonstração de amor e carinho pelo companheiro (F) \\
\hline \multirow{6}{*}{ Função de papéis } & \multirow[t]{4}{*}{ Ansiedade } & Dependência financeira (F) \\
\hline & & Preocupação com o filho e com a manutenção do lar (C) \\
\hline & & Quimioterapia (C) \\
\hline & & Alteração nos papéis (C) \\
\hline & \multirow[t]{2}{*}{ Alteração nos papéis } & Limitação das atividades pelo tratamento quimioterápico (F) \\
\hline & & Sentimento de inutilidade (R) \\
\hline \multirow[t]{8}{*}{ Interdependência } & Dificuldades de inserção em grupos & Maneira de ser ("fechada") (F) \\
\hline & \multirow[t]{3}{*}{ sociais } & Vergonha pela falta da mama (F) \\
\hline & & Mastectomia (C) \\
\hline & & Mudança no local de moradia (F) \\
\hline & \multirow[t]{3}{*}{ Alterações no relacionamento com amigos } & Mastectomia e quimioterapia (C) \\
\hline & & Medo de não ser aceita pelas mudanças na aparência (R) \\
\hline & & Vergonha (F) \\
\hline & Dificuldade para demonstrar carinho e afeição & Retração pela maneira como foi educada (R) \\
\hline
\end{tabular}


sendo então identificados os estímulos decorrentes do tratamento quimioterápico, conforme demonstrado na Tabela 1.

A mastectomia costuma causar impacto à mulher, pois abala a sua auto-estima. Q uando associada à quimioterapia, esse impacto aumenta ainda mais em função dos efeitos colaterais decorrentes, especialmente a queda de cabelo. Silva $\&$ al. ${ }^{10}$ evidenciaram em um estudo que a mulher, frente ao tratamento quimioterápico, apresenta respostas ineficazes que se refletem em medo, depressão, angústia e tristeza.
Diagnósticos de enfermagem, estabelecimento de metas e intervenções

A partir dos diagnósticos estabelecidos pela associação dos modos adaptativos, comportamentos e estímulos, foram elaboradas as intervenções de enfermagem com o intuito de ajudar a cliente a promover respostas adaptativas aos estímulos presentes em sua condição.

D e acordo com o M odelo de Adaptação de Roy, a meta da enfermagem é a promoção de respostas adaptativas em relação aos quatro modos adaptativos. ${ }^{4}$ Assim, tendo por

Tabela 2. Planejamento das intervenções de enfermagem segundo o diagnóstico de enfermagem

\begin{tabular}{|c|c|}
\hline Diagnóstico de enfermagem & Planejamento das intervenções \\
\hline $\begin{array}{l}\text { Distúrbio no padrão de sono relacionado à } \\
\text { dor e modificações no ambiente }\end{array}$ & $\begin{array}{l}\text { Limitar a ingestão de bebidas com cafeína; Planejar com a cliente } \\
\text { alternativas que a ajudem a conciliar o sono, como: banho, ouvir músicas, } \\
\text { leituras, assistir televisão, massagens; Discutir posições que possam ajudá-la a dormir. }\end{array}$ \\
\hline $\begin{array}{l}\text { Dor relacionada a distúrbios secundários a0 } \\
\text { câncer }\end{array}$ & $\begin{array}{l}\text { Oferecer suporte informativo com relaçãa às causas e duração da dor; } \\
\text { Orientar quanto à solicitaçã̃o de prescrição médica de analgésicos; } \\
\text { Explicar as sensaçõos que poderão estar presentes durante o } \\
\text { tratamento quimioterápico; Discutir métodos de alívio da dor: } \\
\text { relaxamento, estimulação cutânea, massagens; Proporcionar à } \\
\text { cliente, oportunidade para discutir seus medos e dificuldades. }\end{array}$ \\
\hline $\begin{array}{l}\text { Nutrição alterada: ingestão menor do que as } \\
\text { necessidades corporais, relacionada à } \\
\text { diminuição da ingesta oral, desconforto da } \\
\text { boca, náuseas, vômitos, secundários ao } \\
\text { tratamento quimioterápico }\end{array}$ & $\begin{array}{l}\text { Explicar a importância da nutrição adequada; Discutir com } \\
\text { a cliente } 0 \text { consumo de alimentos agradáveis de ingerir, } \\
\text { bem como } 0 \text { uso de temperos que melhorem o sabor e } 0 \text { aroma } \\
\text { dos alimentos; Orientar quanto à realização de pequenas refeições } \\
\text { freqüentes para reduzir a sensaçãa de estômago distendido. }\end{array}$ \\
\hline $\begin{array}{l}\text { Integridade da pele prejudicada, relacionada } \\
\text { aos efeitos da quimioterapia sobre as células } \\
\text { epiteliais e basais }\end{array}$ & $\begin{array}{l}\text { Incentivar o uso diário de hidratantes ou óleos na } \\
\text { pele, realizando massagens; } \\
\text { Ensinar a minimizar a perda de cabelo: evitar uso excessivo de xampu, } \\
\text { usar condicionador duas vezes por semana; secar o cabelo delicadamente; } \\
\text { evitar prender o cabelo; usar pente com dentes separados. Incentivar o } \\
\text { uso de óleo no couro cabeludo Limitar o uso de sabonete }\end{array}$ \\
\hline $\begin{array}{l}\text { Ansiedade relacionada à ameaça ao } \\
\text { autoconceito, secundária à mudança no } \\
\text { relacionamento com os amigos e problemas } \\
\text { financeiros }\end{array}$ & $\begin{array}{l}\text { Explicar que após o término do tratamento, as atividades } \\
\text { profissionais podem ser retomadas caso tenham sido } \\
\text { interrompidas; Proporcionar tranqüilização e conforto; } \\
\text { Incentivar a participação em atividades de grupo e sociais; }\end{array}$ \\
\hline $\begin{array}{l}\text { Distúrbio na imagem corporal relacionado às } \\
\text { mudanças na aparência, secundárias à } \\
\text { quimioterapia }\end{array}$ & $\begin{array}{l}\text { Encorajar o contato com amigos e família; Auxiliar na resolução } \\
\text { de alterações provocadas cirurgicamente no corpo; Encorajar a } \\
\text { cliente a partillhar as preocupações, os medos e a percepção } \\
\text { do impacto das mudanças associadas com a quimioterapia } \\
\text { na sua vida; Explicar que o cabelo crescerá novamente, mas } \\
\text { poderá mudar de textura e de cor; Encorajar o uso de perucas } \\
\text { e/ou lenços e turbantes; Discutir as dificuldades que o } \\
\text { cônjuge/amigos podem ter com as mudanças visíveis. }\end{array}$ \\
\hline $\begin{array}{l}\text { Isolamento social relacionado à aparência desfigurada } \\
\text { e ao medo de rejeiç̧ão, secundários ao câncer }\end{array}$ & $\begin{array}{l}\text { Encorajar a expressão dos sentimentos aos outros; Encorajar a visita } \\
\text { dos amigos e das pessoas; significativas e o contato com amigos e família. }\end{array}$ \\
\hline
\end{tabular}


fundamentação Carpenito ${ }^{8}$ e literaturas relacionadas a câncer de mama e quimioterapia, e ainda trabal hos anteriormente realizados, ${ }_{1}^{10}$ foram planejadas as intervenções (Tabela 2).

0 tratamento quimioterápico com todos os seus efeitos colaterais associados costuma gerar medo nas mulheres, pelo fato de afetar sua auto-imagem ou imagem corporal. A interrupção das atividades também pode ocasionar ansiedade, uma vez que a mulher se sentirá dependente de outra pessoa, tanto financeiramente como na realização de atividades rotineiras.

Asintervenções de enfermagem elaboradas de acordo com cada diagnóstico formulado, foram implementadas junto à paciente, com o intuito de auxiliá-la a manifestar comportamentos adaptativos, contribuindo, assim, para a manutenção da sua integridade.

Avaliação das intervenções

As intervenções foram implementadas e avaliadas pelo acompanhamento à mulher, durante quatro visitas domiciliares. Percebeuse que, com o decorrer das visitas, esta apresentou significativa melhora diante dos estímulos decorrentes do tratamento quimioterápico, manifestando comportamentos que demonstraram sua adaptação. A pesar da paciente continuar apresentando distúrbios no padrão desono, devido, principalmente, às dores na região torácica e na região da cirurgia de reconstrução mamária, o problema foi amenizado pelas intervenções. Q uanto à nutrição, evoluiu deforma desejada, procurando se alimentar em intervalos curtos e ingerir alimentos do seu agrado, evitando os que the causavam enjôo. 0 sentimento detristeza frente aos efeitos da quimioterapia, especialmente a alopecia, já não a incomodavam como no início do tratamento, tendo passado a usar de artifícios para melhoria da sua imagem corporal, tais como lenço ou peruca. A visita a amigos e familiares, aos poucos, passou a fazer parte da sua vida, quando anteriormente, era evitado qualquer contato com pessoas, por imaginar terse tornado diferente, com a perda da mama e com o tratamento. A paciente tem a esperança de poder concluir a cirurgia de reconstrução mamária o quanto antes, afirmando que esse desejo é até maior pelas dores que o "peso da mama" acarreta, do que mesmo pela própria aparência. Com relação ao companheiro, passou a demonstrar maior tranqüilidade em relação à situação dos dois. Alguns diagnósticos inicialmente estabelecidos foram revistos e acabaram sendo retirados, em razão da paciente haver demonstrado comportamentos que contribuíram para a manutenção da sua integridade 0 diálogo com a cliente, como com sua família, auxiliou no alcance das metas e modificação dos comportamentos, com a implementação das intervenções. De forma gradual, a cliente evoluiu de maneira satisfatória, apresentando comportamentos adaptativos. Vale ser enfatizado, também, que a expressão de tristeza, choro e inquietação, manifestada no início do estudo, pouco a pouco, foi sendo substituída por satisfação diante do acompanhamento.

\section{CONSIDERAÇÕES FINAIS}

0 processo de enfermagem proposto no M odelo de Adaptação de R oy é relevante, haja vista ter proporcionado a identificação de estímulos presentes na situação em que se encontrava a cliente do estudo, tornando possível o planejamento de intervenções mais específicas àquela situação.

A par disso, facilitou o acompanhamento à cliente, no sentido de oferecer subsídios para o planejamento das intervenções e 0 alcance da meta de enfermagem através da implementação dessas intervenções. 0 acompanhamento à cliente, dentro do seu contexto, possibilitou uma maior efetividade das intervenções, auxiliando a mulher na promoção de respostas adaptativas, contribuindo, assim, para a manutenção de sua integridade.

A aceitação do acompanhamento, tanto por parte da cliente como da família, foi considerada satisfatória, na medida em que contribuiu para a implementação das intervenções.

\section{REFERÊNCIAS BIBLIOGRÁFICAS}

1. Bender C. I mplicações da quimioterapia paraa enfermagem. In: Clark JC, M acgee RF. Enfermagem oncológica: um currícul o básico. Porto Alegre: Artes M édicas; 1997. p. 325 - 35.

2. Simon SD. Câncer da mama: perspectivas futuras do tratamento quimioterápico. In: Faria 
SL, Leme LH S, O liveira Filho JA. Câncer da mama: diagnóstico etratamento. Rio deJ aneiro: M edsi; 1994. p. 253-7.

3. Roy SC, Andrews H A. Essentials of the Roy adaptation model. In: Roy SC, Andrews H A. The Roy adaptation model. 2nd ed. Stamford: Appleaton \& Lange; 1999. p. 29-62.

4. RoySC, AndrewsH A. Thenursing processaccording to the Roy adaptation model. In: R oy SC, AndrewsH A. T he Roy adaptation model. 2nd ed. Stamford: Appleaton \& Lange; 1999. p. 63-9.

5. Lüdck $M$, André M ED A. Pesquisa em educação: abordagensqualitativas. São Paulo: EPU; 1986. p. 11-24.

6. Christensen PJ, K enney JW. Assessment data collection to thefamily client. In: Christensen PJ , Kenney JW. N ursing process: aplication of conceptual models. 4th ed. St. Louis: M osby; 1995. p. 70-7.

7. Iyer PW,Taptich BJ, Bernocchi-LoseyD .0 processo diagnóstico. In: Iyer PW, Taptich BJ, BernocchiLosey D. Processo ediagnóstico em enfermagem. Porto Alegre: ArtesM édicas; 1993. p. 57-72.

8. Carpenito LH. M anual de diagnósticos em enfermagem. 6aed. Porto Alegre: ArtesM édicas Sul; 1999. 488p.

9. Iyer PW, Taptich BJ, Bernocchi-Losey D. Escrevendo um diagnóstico. In: Iyer PW, Taptich BJ, Bernocchi-Losey D. Processo e diagnóstico em enfermagem. Porto Alegre: Artes M édicas; 1993. p. 75-98.

10. Silva RM , M elo EM , Rodrigues M SP. Família como suporte para a mulher em tratamento quimioterápico. Rev Fam Saúde D esenvol 1999;1(1/2):87-96.

\begin{abstract}
ANEXO

\section{Relato do caso}

M.L.T.L.E., sexo feminino, 40 anos, 10 grau completo, união consensual, aposentada (mas trabalha com vendas), renda familiar aproximadamente $\mathrm{R} \$ 300,00$. Um filho com oito anos, procedente de Fortaleza - Ceará. $M$ astectomizada unilateralmente à esquerda há cinco anos, diagnosticada metástase pulmonar à direita há dois meses, tendo sido indicado o tratamento quimioterápico, já realizado anteriormente. M etástase descoberta a partir de dores na região do tórax posterior, sendo confirmada por um exame de raio $X$ pulmonar ao se submeter a cirurgia de reconstrução mamária. Refere não sentir disposição para as atividades diárias (domésticas, profissionais ou caminhadas) em decorrência do tratamento quimioterápico. Fato que é influenciado também devido ao pouco tempo dedicado ao sono/repouso, visto que costuma dedicar aproximadamentequatro horas ao sono no período de 24 horas, devido a presença de dores na região torácica posterior, tipo "ferruada" e na região da cirurgia de reconstrução mamária, tornando difícil conciliar bem o sono. Durante o dia não costuma dormir. $\mathrm{N}$ ão utiliza nenhum tipo de auxílio a este problema. Tônus muscular diminuído, ausência de atrofias e alterações

na postura e modo de andar. Realiza cerca de seis refeições diárias, pois se ficar muito tempo sem comer apresenta "suor frio". Ingere grande quantidade de líquidos durante o dia. Refere redução de oito quilos no peso, tendo sido citado anorexia e enjôo pela quimioterapia, como também náuseas. Eliminações intestinais e urinárias presentes, com quantidade, cor e odor característicos. Respiração abdominal, padrão normal, refere tosse e dor torácica. Geralmente sente frio, sem história de doenças crônicodegenerativas. Sinais vitais normalizados. Amenorréia, após realização da reconstrução mamária. $\mathrm{N}$ ão apresenta fatores de risco para carcinoma mamário. Realiza o auto-exame da mama mensalmente, em várias posições (sentada, deitada, em pé). Pele íntegra, coloração e textura dentro dos padrões de normalidade, porém ressecada. $M$ embranas mucosas normocoradas e preservadas, alopécia quase total, couro cabeludo ressecado e apresentando pequenas bolhas e prurido. Acuidade auditiva um pouco diminuída, uso de óculos para leitura, sensibilidade gustativa etátil presentes. Estado geral bom, consciente, orientada. Apresenta ansiedade, relacionada com problemas financeiros. Refere medo de andar sozinha, visto "estar fraca" depois da
\end{abstract}


quimioterapia. Sente-se satisfeita consigo mesmo. Alterações na vida após a mastectomia: mudança no relacionamento com o companheiro, pois este não demonstra mais carinho e afeição à cliente, tornou-se mais distante. Alterações na vida pela quimioterapia: o companheiro afastou-se ainda mais, e ela própria se isolou das pessoas pela vergonha da queda do cabelo e pela curiosidade manifestada. Sente-se dependente dos outros em todos os sentidos, no plano econômico, na realização de atividades simples e até para sair. Costuma expressar sua percepção/pensamento através de gestos, ações, diálogos e troca de idéias, ressaltando que após a quimioterapia ficou mais "fechada", mais distante das pessoas. Percebe-se como uma pessoa calada, eno grupo social demora um pouco para ser aceita. Frente ao retorno ao tratamento quimioterápico, a família ficou profundamente triste e receosa. H ouve alterações nos papéis dentro da família e sociedade após a quimioterapia, pelo fato de ter parado de trabal har e depender dos outros. A relação com os familiares é considerada boa, estes preocupam-se com a saúde da cliente e procuram ajuda-la financeiramente. 0 relacionamento com os amigos ficou alterado após a mastectomia, pois teve que mudar de residência e de emprego. Com relação à quimioterapia, o relacionamento com os amigos modificou-se um pouco, pois mudouse novamente e tem vergonha da queda de cabelo. N ão se sente à vontade para demonstrar carinho e afeição as pessoas, mas as pessoa demonstram essas atitudes para com ela, através do toque, sorriso, diálogo. As mudanças após o retorno ao tratamento quimioterápico referem-seao fato deter parado de trabal har, não realizar atividades domésticas, não realizar atividades aeróbicas (caminhadas) e dependência dos outros. Diante disso sentese inútil e triste. 